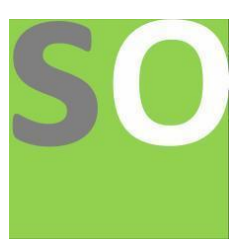

Article title: CoViD-19: An early intervention therapeutic strategy to prevent developing a severe disease as an alternative approach to control the pandemic

Authors: HAMID MERCHANT[1]

Affiliations: University of Huddersfield, United Kingdom[1]

Orcid ids: 0000-0002-7668-5013[1]

Contact e-mail: hamid.merchant@hud.ac.uk

License information: This work has been published open access under Creative Commons Attribution License http://creativecommons.org/licenses/by/4.0/, which permits unrestricted use, distribution, and reproduction in any medium, provided the original work is properly cited. Conditions, terms of use and publishing policy can be found at https://www.scienceopen.com/.

Preprint statement: This article is a preprint and has not been peer-reviewed, under consideration and submitted to ScienceOpen Preprints for open peer review.

Funder: Not Applicable

DOI: 10.14293/S2199-1006.1.SOR-.PPURWMT.v1

Preprint first posted online: 23 May 2020

Keywords: CoViD-19, Coronavirus, SARS-CoV-2, Early Intervention, CoViD19, SARS-CoV, Essential Oils, Oropharyngeal sanitisation, Steam, Pandemic 


\title{
CoViD-19: An early intervention therapeutic strategy to prevent developing a severe disease as an alternative approach to control the pandemic
}

\author{
"Hitting the coronavirus hard before it causes a severe disease" \\ Dr Hamid A. Merchant ${ }^{1}$ \\ Department of Pharmacy, School of Applied Sciences, University of Huddersfield, Huddersfield \\ HD1 3DH, West Yorkshire, United Kingdom
}

\begin{abstract}
While we wait for a confirmed drug or a vaccine for CoViD-19, it may be possible to intervene early to prevent the virus causing a severe disease to offer an alternative therapeutic strategy to control the pandemic. The global burden of CoViD-19 on healthcare system can be significantly reduced by targeting CoViD-19 patients with or without symptoms who are selfisolating at home or in a quarantine. If a therapeutic support can be offered to this group of patients that could attenuate the virus within the upper respiratory tract during the early stages of CoViD-19, it can give the body the time to produce enough antibodies to recover naturally from the disease before progressing into a severe disease. An early intervention can, therefore, prevent the virus to get down the lower respiratory tract, reduce the number of cases with severe disease involving pneumonia and the need for hospitalisation. This article presents a simple yet holistic treatment strategy that involves inhaling steam supplemented with essential oils possessing wide spectrum antimicrobial properties in conjunction with oropharyngeal sanitisation to all those who are CoViD-19 positive or are under self-isolation due to symptoms. The approach is very simple, cheap, and effective in relieving the symptoms of the disease and is likely to reduce the viral load in the upper respiratory tract that may help recover from the infection. Since there is no vaccine or treatment yet approved to prevent or treat the CoViD-19, the importance of early intervention is invaluable in reducing the global disease burden. In author's opinion, this strategy may be very effective to nip the infection in the bud before it gets difficult to treat and therefore, have a potential to significantly reduce the CoViD-19 associated hospitalisation.
\end{abstract}

Keywords: CoViD-19; Coronavirus; SARS-CoV-2; Early Intervention; CoViD19; SARS-CoV

\section{Expert Opinion}

The therapeutic strategy involves on an early intervention approach, 'hit the virus hard before it takes control'. The treatment involves using inhaled steam supplemented by essential oils possessing natural antimicrobial properties, oropharyngeal sanitisation and remedies for symptomatic relief. Inhalation of steam supplemented with antimicrobial essential oils may help attenuate the virus in nasal cavity, nasopharynx, oropharynx, and laryngopharyx, antiseptic mouthwashes and gargles can help sanitise oral cavity and oropharynx, whereas antiseptic lozenges can help sanitising oro- and laryngopharynx. The gastrointestinal symptoms can be managed with over the counter remedies to regulate the bowel.

The strategy provides a holistic pharmaceutical care plan to support patients with mild to moderate CoViD19

\footnotetext{
${ }^{1}$ Tel: +44 1484 472387, Email: hamid.merchant@hud.ac.uk
} 
until an approved drug or vaccine becomes available. Figure 1 graphically illustrates the treatment strategy outlined in this article.

\section{Stream supplemented by the essential oils}

It is widely known that inhaling steam can help relieving symptoms of respiratory illnesses, such as the cold and flu. Historically, it has been an effective way to help patients recover from pneumonia before the antibiotics were abundant. Steam can help to clear the virus by mobilising the mucus. Moreover, the treatment can also heat attenuate the virus by a direct steam action. The heat attenuation is a common practice in microbiology and often used in preparing live attenuated vaccines. The attenuated viruses are less pathogenic and could give body the time to produce antibodies and recover naturally. The steam treatment has a long history of safe human use, it helps in mitigating symptoms, and also accelerates the recovery from various respiratory illnesses. The treatment is simple and cheap and can be taken in a household. There are various facial steamers available in the market, food steamers, electric kettles, and even a classic steaming hot water in a bowl can also do the job.

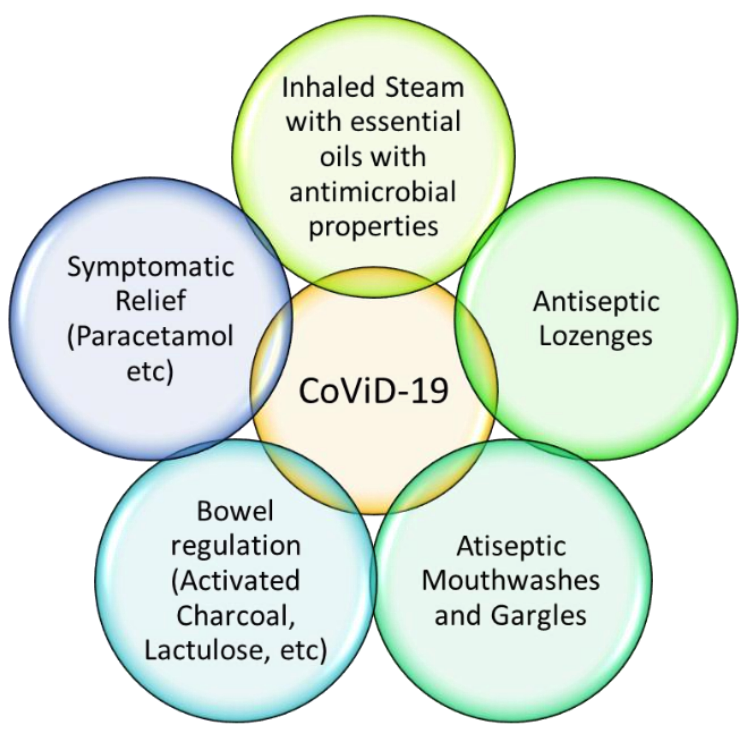

Figure 1. Therapeutic intervention for the management of early CoViD-19
It is recommended that steam should be supplemented by the essential oils (aromatherapy oils) that naturally possess the antimicrobial properties. The steam will carry the tiny fumes of the antimicrobial constituents from these essential oils into the respiratory tract and is likely to improve the efficacy of the steam treatment. This is very similar to taking a medicine through a nebuliser in a clinic. Unlike a nebuliser where a gas is used to inhale the medicine, this technique uses the steam.

The steam supplemented by antimicrobial volatile oils may help providing a local antimicrobial effect within the airways. There is enough laboratory evidence in support of the antimicrobial properties of various essential oils [1-7]. The essential oils have been reported to possess a broad spectrum antimicrobial properties against various pathogenic bacteria, fungi, and viruses, and also drug resistant organism [1-6]. Recently, an in-silico modelling suggests that essential oil components may interact with key protein targets of SARSCoV-2 [7]. We do not have a direct evidence on SARS-CoV-2 as yet, albeit it is highly likely that essential oils can be effective over a broad spectrum of viruses due to their mechanism of action. The list below are the essential oils that have a safeuse history in human and may therefore be recommended for treatment at this stage. There are a variety of other essential oils that seems to have stronger properties in scientific literature [1-7] but cannot all be recommended as yet due to the lack of safety data for human use by inhalation. The list below can be updated based on further evidence and research that is currently being published at the breakneck speed.

- Peppermint oil
- Clove (bud) oil
- Eucalyptus oil
$\circ$ Camphor oil,
$\circ$ the oil of Thyme, and
$\circ$ the Cinnamon leaf oil

- Peppermint oil

- Clove (bud) oil

- Eucalyptus oil

- Camphor oil

- the Cinnamon leaf oil 
Just a drop of these oils (each) added to the aromatherapy dispenser during the steam treatment should be sufficient. These oils are easily available via online retailers and are not expensive at all.

These oils have traditionally been used in vapour rubs, nasal sticks etc., for use in common cold and flu, they are much safer than various other oils reported in the literature due to their safe-use history by inhalation in humans. They have a very strong antimicrobial property if inhaled directly with a hot steam. One of the very old product in the market is a Vick's Vaporub [8] that had been added to the steaming water historically to help recover from the viral illness of the respiratory tract. Therefore, if public cannot find these oils easily, they can use a dab of the vapour rub in the steaming hot water. Vicks ${ }^{\circledR}$ Vaporub contains camphor, menthol, eucalyptus, cedarleaf oil, nutmeg oil, thymol and turpentine oil [8]. The vapour rubs do not contain all of the essential oils suggested above and their recipe may vary with the brand but many includes a good mix that can provide an easy alternative to find the individual oils. The history of using essential oils in a vapour rub format is very old and dates back to 1890's and attributed to Lunsford Richardson, a pharmacist from North Carolina who created Vicks ${ }^{\circledR}$ home remedies. During 1918-19 Spanish flu, Vicks ${ }^{\circledR}$ Vaporub was then a very popular product and widely used and recommended to help relieve the deadly flu symptoms [9]. The addition of vaporubs into a steaming water for inhalation can be messy due to the presence of the greasy ointment base (soft paraffin or petrolatum). There is also a dispersible shower tablet available from Vicks ${ }^{\circledR}$ that contains similar composition of essential oils qualitatively [10]. This can be added to the steaming hot water easily for the purpose of this treatment by inhalation.

\section{Oropharyngeal sanitisation}

There are many products available in the market that can help to provide a local disinfection of the oral cavity and the back of the throat. Some of the examples are listed below which can be very helpful to reduce the viral load in the oral cavity and may prevent the disease progression to the lower respiratory tract. This should be used in conjunction with the steam based treatment detailed above.

Lozenges

2,4-dichlorobenzyl alcohol and amylmetacresol lozenges such as those available as Strepsils ${ }^{\circledR}$ provides a strong antiseptic property locally in the throat [11]. The combination of dichlorobenzyl alcohol and amylmetacresol gives a wide spectrum antiseptic properties against a range of bacteria and viruses. A study in 2005 even have shown this combination to effective against respiratory syncytial virus, influenza A and SARS-CoV-1 [12].

\section{Mouthwashes}

There are plenty of mouthwashes and gargles with a very good antimicrobial and anti-inflammatory properties that can be helpful as an adjunct. These mouthwashes can also be used as gargles to target the virus infiltrating the throat lining

- Benzydamine mouthwash or the throat rinse (contains analgesic and antiinflammatory properties) [13]

- Chlorhexidine digluconate antiseptic mouthwash (provides an antimicrobial activity against most of the pathogens of oral cavity including bacteria, yeasts, fungi and viruses) [14]

Albeit less effective than above, but a cheaper alternative to the above may include the following. This can provide an alternative if cost or product availability is a concern amid CoViD-19.

- Warm saline water (a gargle with warm salty water is a classic antiseptic for the throat)

- A gargle with warm water with dispersible aspirin can provide analgesic and anti-inflammatory properties to help with the throat inflammation) 


\section{Bowel regulation}

The coronavirus is also known to cause upset stomach and many patients may present with symptoms of diarrhoea or frequent bowel openings, this is due to the gastroenteritis caused by the virus. The virus may be ingested through contaminated food (uncooked food, like salads etc) or may end up into the gut by swallowing their own saliva or the mucus by the patient. There have been a few studies which measured the presence of SARS-CoV-2 in various body fluids of the infected patient, such as saliva, sputum, blood, urine and stools $[15,16]$. The virus has been reported to be present in the stool samples of the patients for several days even after the recovery from the infection. A poor hygiene, poor sanitation, and contaminated water may, therefore, also spread the virus along with airborne transmission. The study also provides a case series from China with evidence for a gastrointestinal infection caused by the SARS-CoV-2 [17]. Moreover, the virus form the gut may also get through to the liver and can make the disease more severe.

One of the strategy that may help to clear the virus from the gut is the use of activated charcoal [18], ispaghula husk [19], or the Movicol $^{\circledR}$ (containing a polymer macrogol) [20]. These products are termed as 'bulkforming laxatives', they swell in the presence of water and helps with constipation. If a concentrated form of the product is taken (less water) then they have a tendency to adsorb the water from the gut to help with the diarrhoea. They have also been reported to absorb pathogens from the gut due to their high surface area. Their scavenging property may sweep over the virus form the gut and help cleanse the bowel. To the least, the strategy will help patients symptomatically with irregular bowel movements. If gastrointestinal spasm is an issue, a combination product with an antispasmodic such as mebeverine can be offered [21].
Conversely, if patients may present with constipations, they can should be advised to take ispaghula or Movicol ${ }^{\circledR}$ with plenty of warm water to help regulating the bowel. They may also be offered other common laxatives, such as Senokot ${ }^{\circledR}[22]$ or lactulose [23] to regulate their bowel and help clearing the virus from the gastrointestinal tract.

\section{Symptomatic Relief}

The patients should also be provided other symptomatic relief as the case may be. This may include a regular dose or paracetamol to help with the fever and analgesia, may include antihistamines, cough syrup and other over the counter remedies individualised to their need. The patient may need reliever inhalers or nebulisers if breathing difficulty is an issue. A spacer device should be considered amid CoViD19 to ensure adequate inhalation of the emitted dose. The patient with asthma or COPD should be advised to continue their regular inhalers including steroids (minimum possible dose) to prevent any exacerbations.

\section{Conclusion}

Often, the solution to worlds most complicated issues like CoViD-19 can be so simple that the human mind finds it very difficult to accept.

It is hoped that if we can support those with mild to moderate CoViD-19 symptom to help them recover at home, we can significantly reduce the disease burden on health systems and free up more space for those who are at higher risk of developing a serious disease, for instance elderly, the immunocompromised patients or those with other pre-existing conditions, or those who are waiting for a treatment for other medical conditions with significant impact on their quality of life.

Table 1 summarises the treatment strategy that can be used by healthcare 
professionals fighting in the front line to save lives. The community pharmacists who are already a first port of call for many patients can be instrumental in the therapeutic management of CoViD-19 at the early stages.

Table 1. Therapeutic intervention for the management of early CoViD-19 by the frontline healthcare professionals.

\section{Therapeutic agent Description}

Steam supplemented by essential oils

Antiseptic lozenges

Antiseptic mouthwashes or gargles

Bowel regulation compliance.
Add a drop each from peppermint oil, clove (bud) oil, eucalyptus oil, camphor oil, the oil of thyme, and the cinnamon leaf oil to the steaming hot water. Steam can be inhaled three to four times daily depending on the severity of symptoms. For mild to no symptoms, once daily at bed time will also be useful. A facial steamer or other electric devices can also be used, however, it is advised not to add oils directly into the water reservoir.

A dab of commonly available Vaporub such as Vicks ${ }^{\circledR}$ ore equivalent can also be used if individual oils are difficult to obtain amid pandemic.

A newer dispersible shower tablet is also available in the market from Vicks ${ }^{\circledR}$ that may be added to the water to inhale steam.

2,4-dichlorobenzyl alcohol and amylmetacresol containing lozenges such as Strepsils ${ }^{\circledR}$ or equivalent may be recommended. The combination of these antiseptic compounds gives a wide spectrum antiseptic properties against a range of bacteria and viruses including SARS-CoV-1; efficacy on SARS-CoV2 is not yet known but is likely.

Chlorhexidine digluconate antiseptic mouthwash or gargles will provide an antimicrobial activity against most of the pathogens of oral cavity including bacteria, yeasts, fungi and viruses.

Benzydamine containing mouthwash or the throat rinse can also be supplemented to offer additional analgesic and anti-inflammatory properties. Chlorhexidine and benzydamine are also available as combination product in some countries and may improve patience

A cheaper alternative to the above may include regular gargle with warm salty water which serves is a classic antiseptic. A dispersible aspirin tablet can also be added to the warm water and gargled once or twice a day to offer analgesic and anti-inflammatory properties. This can provide an alternative if cost or product availability is a concern amid CoViD-19.

There is a good evidence to suggest that virus also infects the gastrointestinal (GI) tract of the CoViD-19 patients. Therefore, patient may present with GI symptoms and clearing off the virus from gut is also an important therapeutic consideration. Offer activated charcoal, ispaghula husk, or Movicol ${ }^{\circledR}$ in concentrated form if patients present with diarrhoea. In case of gastrointestinal spasm, an antispasmodic agent such as hyoscine or mebeverine may be added.

Conversely, if a patient suffers from constipation, suggest a very diluted form of ispaghula husk, Movicol ${ }^{\circledR}$ in warm water. Other remedies such as senna (Senokot ${ }^{\circledR}$ ), or lactulose may also be advised to regulate the bowel.

Others device) device) etc as the case may be. A regular use of paracetamol may be preferred in all patients. 


\section{References}

[1] Aleksic Sabo V, Knezevic P. Antimicrobial activity of Eucalyptus camaldulensis Dehn. plant extracts and essential oils: A review. Ind Crops Prod. 2019 Jun;132:413-429. doi: 10.1016/j.indcrop.2019.02.051. [2] Trong Le N, Viet Ho D, Quoc Doan T, Tuan Le A, Raal A, Usai D, Sanna G, Carta A, Rappelli P, Diaz N, Cappuccinelli P, Zanetti S, Thi Nguyen H, Donadu MG. Biological Activities of Essential Oils from Leaves of Paramignya trimera (Oliv.) Guillaum and Limnocitrus littoralis (Miq.) Swingle. Antibiotics (Basel). 2020 Apr 24;9(4). pii: E207. doi: 10.3390/antibiotics9040207.

[3] Knezevic P, Aleksic V, Simin N, Svircev E, Petrovic A, Mimica-Dukic N. Antimicrobial activity of Eucalyptus camaldulensis essential oils and their interactions with conventional antimicrobial agents against multi-drug resistant Acinetobacter baumannii. J Ethnopharmacol. 2016 Feb 3;178:125-36. doi: 10.1016/j.jep.2015.12.008.

[4] Schnitzler P. Essential Oils for the Treatment of Herpes Simplex Virus Infections. Chemotherapy. 2019;64(1):1-7. doi: 10.1159/000501062.

[5] Tariq S, Wani S, Rasool W, Shafi K, Bhat MA, Prabhakar A, Shalla AH, Rather MA. A comprehensive review of the antibacterial, antifungal and antiviral potential of essential oils and their chemical constituents against drug-resistant microbial pathogens. Microb Pathog. 2019 Sep;134:103580. doi:10.1016/j.micpath.2019.103580.

[6] Brochot A, Guilbot A, Haddioui L, Roques C. Antibacterial, antifungal, and antiviral effects of three essential oil blends. Microbiologyopen. 2017 Aug;6(4). doi: 10.1002/mbo3.459. Epub 2017 Mar 14.

[7] Silva JKRD, Figueiredo PLB, Byler KG, Setzer WN. Essential Oils as Antiviral Agents. Potential of Essential Oils to Treat SARS-CoV-2 Infection: An In-Silico Investigation. Int J Mol Sci. 2020 May 12;21(10). pii: E3426. doi: $10.3390 /$ ijms 21103426.

[8] Vicks vaporub, https://vicks.com/en-us/shopproducts/vaporub/vicks-vaporub-topicalcough-suppresant

[9] Vicks History, https://vicks.com/en-us/vickshistory

[10] Vicks VapoShower, https://vicks.com/enus/shop-products/vaporub/vicks-vaposhoweraromatherapy-shower-bomb-soothing-vicksvapor-steam

[11] Strepsils Honey and Lemon, https://www.medicines.org.uk/emc/product/ 5606/smpc
[12] Oxford JS, Lambkin R, Gibb I, Balasingam S, Chan C, Catchpole A. A throat lozenge containing amyl meta cresol and dichlorobenzyl alcohol has a direct virucidal effect on respiratory syncytial virus, influenza $A$ and SARS-CoV. Antivir Chem Chemother. 2005;16(2):129-134. doi:10.1177/095632020501600205

[13] Difflam Throat Rinse, https://www.medicines.org.uk/emc/product/ 9258/smpc

[14] Corsodyl Mouthwash, https://www.medicines.org.uk/emc/product/ $529 / \mathrm{smpc}$

[15] Zheng S, Fan J, Yu F, Feng B, Lou B, Zou Q, Xie G, Lin S, Wang R, Yang X, Chen W, Wang Q, Zhang D, Liu Y, Gong R, Ma Z, Lu S, Xiao Y, Gu Y, Zhang J, Yao H, Xu K, Lu X, Wei G, Zhou J, Fang Q, Cai H, Qiu Y, Sheng J, Chen Y, Liang T. Viral load dynamics and disease severity in patients infected with SARS-CoV-2 in Zhejiang province, China, January-March 2020: retrospective cohort study. BMJ. $2020 \mathrm{Apr}$ 21;369:m1443. doi: 10.1136/bmj.m1443.

[16] Chen C, Gao G, Xu Y, Pu L, Wang Q, Wang L, Wang W, Song Y, Chen M, Wang L, Yu F, Yang S, Tang $Y$, Zhao L, Wang H, Wang $Y$, Zeng H, Zhang F. SARS-CoV-2-Positive Sputum and Feces After Conversion of Pharyngeal Samples in Patients With COVID-19. Ann Intern Med. 2020 Mar 30. doi: 10.7326/M20-0991.

[17] Xiao F, Tang M, Zheng X, Liu Y, Li X, Shan H. Evidence for Gastrointestinal Infection of SARSCoV-2. Gastroenterology. 2020 May;158(6):18311833.e3. doi:10.1053/j.gastro.2020.02.055.

[18] Carbomix (activated charcoal). https://www.medicines.org.uk/emc/product/ 238

[19] Fybogel granules, https://www.medicines.org.uk/emc/product/ $618 / \mathrm{smpc}$

[20] Movicol sachet, https://www.medicines.org.uk/emc/product/ 1025/smpc

[21] Fybogel Mebeverine Granules for oral suspension, https://www.medicines.org.uk/emc/product/ 1099/smpc

[22] Senokot tablets, https://www.medicines.org.uk/emc/product/ 11140/smpc

[23] Duphalac soluiton, https://www.medicines.org.uk/emc/product/ $5525 / \mathrm{smpc}$ 


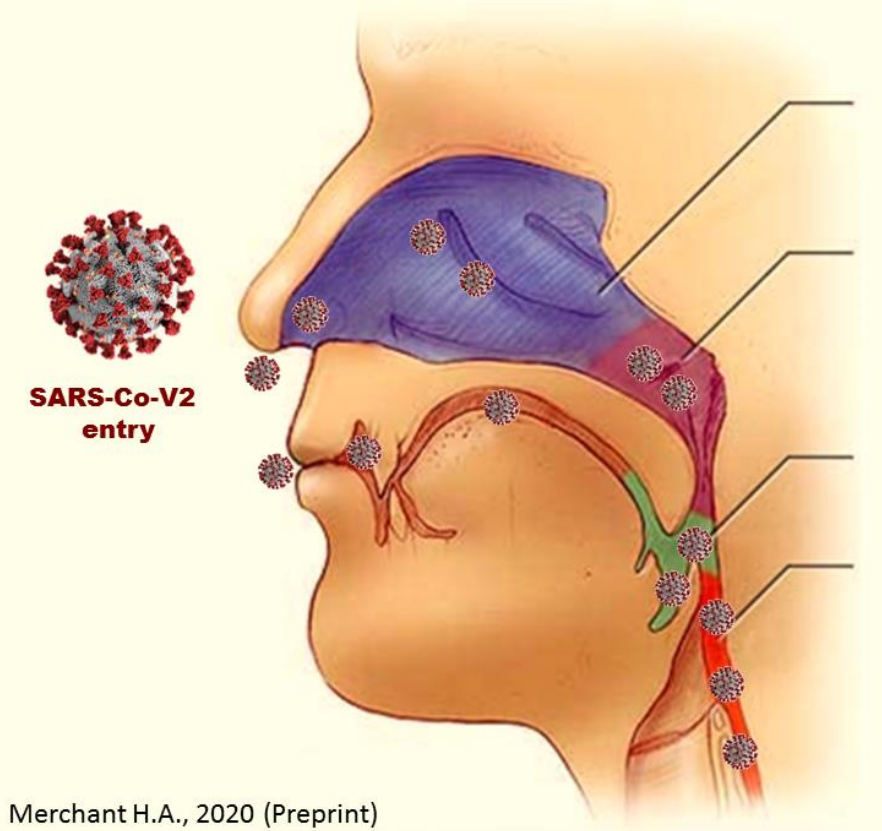

Nasal Cavity

Inhaled Steam supplemented with Antimicrobial Essential Oils

Nasopharynx

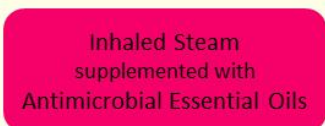

Inhaled Steam with Antimicrobial Essential Oils

\section{Oropharynx}

$\stackrel{+}{+}$ Antiseptic Mouthwashes

Antiseptic Gargles

Antiseptic Lozenges

Laryngopharynx

Inhaled Steam with Antimicrobial Essential Oils $+$

Antiseptic Lozenges

Merchant H.A., 2020 (Preprint) 